\title{
Positron scattering from neon and argon
}

\author{
A. C. L. Jones, C. Makochekanwa, P. Caradonna, D. S. Slaughter, J. R. Machacek, R. P. McEachran, \\ J. P. Sullivan, and S. J. Buckman* \\ ARC Centre for Antimatter-Matter Studies, Research School of Physics and Engineering, Australian National University, \\ Canberra 0200 Australia
}

A. D. Stauffer

Department of Physics, York University, Toronto, Canada

I. Bray and D. V. Fursa

ARC Centre for Antimatter-Matter Studies, Curtin University of Technology, G.P.O. Box U1987, Perth, Western Australia 6845, Australia

(Received 28 November 2010; published 3 March 2011)

\begin{abstract}
High-resolution measurements of positron interactions with $\mathrm{Ne}$ and Ar are presented, as well as theoretical treatments. The data extend over a range of 0.3 to $60 \mathrm{eV}$ and comprise measurements of the grand total, positonium formation, and grand total minus positronium formation cross sections. Theoretical treatments of scattering from $\mathrm{Ne}$ and $\mathrm{Ar}$ are performed under the relativistic optical potential approach, as well as calculations using the convergent close-coupling method. Comparisons of the present measurements and theories are made with previous theoretical and experimental work.
\end{abstract}

DOI: 10.1103/PhysRevA.83.032701

PACS number(s): 34.80.Uv, 34.80.Bm, 36.10.Dr

\section{INTRODUCTION}

The investigation of positron scattering from noble gases provides a vital meeting point for contemporary quantum scattering theory and experiment. The noble-gas systems are some of the simplest targets to study experimentally due to their inert nature and consequent ease of handling. With their closed valence shells they are also relatively simple collision systems to approach theoretically, although the level of complication increases with atomic number. In this work we aim to provide benchmark experimental cross sections for atomic rare-gas targets that are more complex than helium. Through a detailed comparison of this data with recent theoretical treatments, we also hope to critically evaluate both experiment and theory with a view to establishing an accepted set of cross sections for these gases.

A sizable body of previous work exists for both $\mathrm{Ne}$ and Ar, with most of the measurements being carried out from the early 1970s up until the mid-1980s. Despite more than half a dozen groups independently investigating these systems, considerable disparity remains, even for measurements of the total cross section, though considerable effort was made to understand possible sources of systematic errors [1]. Many improvements have been made in both the techniques employed and the equipment available, such that this problem may be better approached now. The broader intent in understanding these relatively simple scattering cases, both experimentally and theoretically, is to further refine our understanding of positron interactions in general, and thus eventually allow theoretical treatment of problems which cannot easily be tackled experimentally. Examples of such systems might be large molecules of biological interest that may play a role in positron emission tomography, a medical imaging technology. See, for example [2], and references therein. In the following

\footnotetext{
*stephen.buckman@anu.edu.au
}

sections we explain briefly both our experimental and theoretical approaches, before giving a detailed comparison between present experiment and theory with that which has preceded us. We conclude with a summary and some future plans.

\section{EXPERIMENTAL DETAILS}

High-resolution measurements of total cross sections have been made for positron scattering from neon and argon. The measurements were performed using a "Surko" buffer-gas trap and beam apparatus. The design of the experiment has been covered in detail previously [3] and will be only briefly discussed here. Positrons were generated from a $50 \mathrm{mCi}^{22} \mathrm{Na}$ source and moderated with a solid Ne moderator, producing a continuous beam with a $1.5 \mathrm{eV}$ energy spread and a current of $0.5-1 \mathrm{pA}$. Using an axially confining magnetic field $(\sim 100 \mathrm{G})$, the moderated positrons are guided electrostatically to the trap, which consists of two pressure stages, with $\mathrm{N}_{2}$ and $\mathrm{CF}_{4}$ buffer gases. The effective throughput efficiency of the trap is approximately $5 \%$, resulting in an average, pulsed current of around $50 \mathrm{fA}$. Under typical operating conditions the trap is cycled at $80-100 \mathrm{~Hz}$ and each pulse contains about 1000-4000 positrons. The energy resolution for these experiments varied in the range $50-80 \mathrm{meV}$. Within the trap, and downstream from it, the pulsed beam is confined by a $530 \mathrm{G}$ magnetic field as it enters a 20-cm-long scattering cell containing the rare-gas target. The large magnetic field ensures that all positrons, except for those lost to positronium formation, scattered and unscattered, are transmitted through the scattering cell before being energy analyzed in a retarding potential analyzer (see [3] for details) and detected on a multichannel-plate detector.

The basic principle behind the total cross section measurements in positron scattering is the transmission method. By use of the Beer-Lambert law, a knowledge of the fraction of the incident current which is transmitted through the scattering 
cell, as well as a measurement of the target gas pressure and the physical length over which the interaction occurs, are sufficient to calculate the total scattering cross section. One of the most significant common problems in measuring total cross sections is in distinguishing between the unscattered incident beam and positrons that undergo small energy loss inelastic and/or scattering at small forward angles.

Details of the analysis techniques used in the present experiment have been covered previously [4,5]. In the present experiment the problem of miscounting small-angle-scattering events is limited by the energy resolution of the incident beam and is defined by the potential applied to the retarding potential analyzer (RPA) positioned between the scattering cell and the detector. This problem has been discussed in a recent paper [6] but for completeness we will repeat the essential details here. The characteristic angle $\theta_{\text {min }}$ below which scattered positrons cannot be distinguished from the incident beam tends to zero for increasing impact energy and can be simply calculated as follows:

$$
\theta_{\min }=\sin ^{-1}\left(\sqrt{\frac{e \Delta V}{E_{S C}}}\right)
$$

where $\Delta V$ is the difference between the potential that cuts off half the beam and the potential at which $I_{m}$, the transmitted intensity, is measured. $E_{S C}$ is the positron incident energy in $\mathrm{eV}$ and $e$ is the elementary charge, equal to 1 when working in units of electron volts.

In these experiments the transmitted fraction is measured with the aid of an RPA, with gas present in the cell. The RPA discriminates against the axial component of the energy of the beam, which is diminished in the event of scattering inelastically or scattering through some angle, and thus provides a simple measurement of the total scattering. This method still misses some fraction of the forward-angle elastic scattering, though in contrast to traditional experiments, this problem becomes smaller with increasing energy. In many of the prior experimental arrangements the ability to discriminate against small forward-angle scattering was limited by geometrical concerns. The role of this common limitation with respect to cross-section measurements has been explored in further detail in a recent publication by our group [6]. In our apparatus this limitation is determined by the resolution of the beam and is typically a small fixed potential offset $(\leqslant 150 \mathrm{mV})$. On the down side, the angles missed also include those scattered backward at close to $180^{\circ}$ due to the reflection of the backscattered positrons and their subsequent detection.

All of the information required to determine the grand total $\left(\sigma_{G T}\right)$ and positronium (Ps) formation $\left(\sigma_{P s}\right)$ scattering cross sections from this experiment are contained in the RPA transmission curve, a schematic example of which is shown in Fig. 1. Each point on the RPA curve is a measure of the number of transmitted positrons as a function of the RPA potential. $I_{0 R}$ is a measurement of the full incident intensity with the energy in the scattering cell set below the Ps formation threshold $\left(E_{P S}\right)$ and the RPA set to transmit all positrons, scattered and unscattered. $I_{0}$ is the measure of the transmitted intensity at the desired scattering energy, and the difference between $I_{0}$ and $I_{0 R}$ is the proportion of positrons that form positronium inside the scattering cell. $I_{0}^{\prime}$ is measured just before the point that

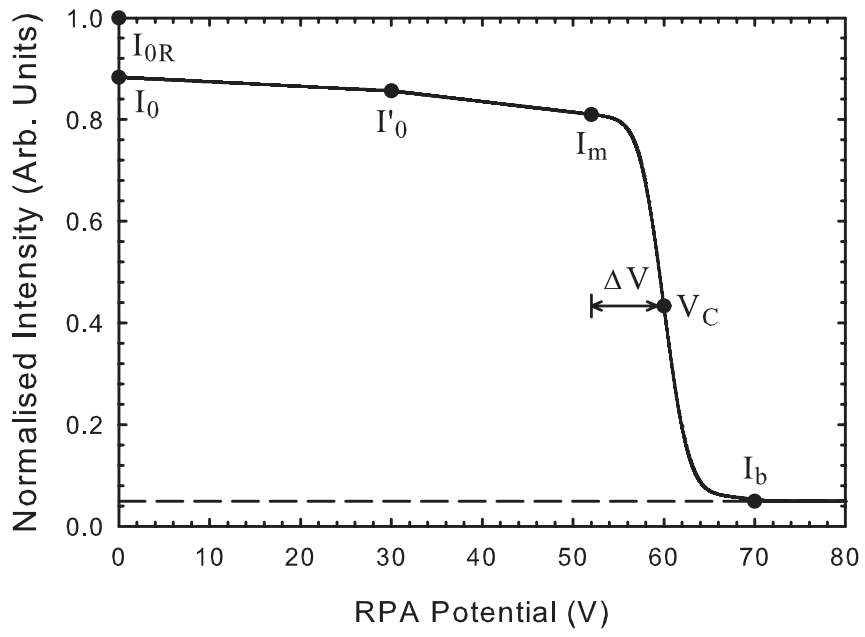

FIG. 1. Schematic example of an RPA transmission curve (see text for details).

corresponds to the onset of any scattering process (taking into account both inelastic and angular scattering). Any difference between $I_{0}$ and $I_{0}^{\prime}$ is indicative of positron scattering outside the gas cell, and is carefully accounted for in the analysis and included in the overall determination of errors in the cross section. $I_{m}$ is determined by setting the RPA to a potential at a small fixed voltage offset [4] from the cutoff potential $\left(V_{C}\right)$ and provides a measure of the unscattered intensity. $I_{b}$ measures the background signal above the RPA cutoff point of the positron detection system.

The grand total cross section $\sigma_{G T}$ is therefore given by

$$
\sigma_{G T}=\frac{1}{n_{m} l} \ln \left(\frac{I_{0 R}}{I_{m}}\right) .
$$

The partial cross sections can then be derived from the total cross section based on the fraction of scattering attributed to each process. These fractions are described by the ratios $R_{P s}$ and $R_{G T-P s}$ for Ps formation and the grand total minus Ps formation, respectively. The fraction of Ps formation scattering is determined from the following ratio of measured intensities:

$$
R_{P s}=\frac{I_{0 R}-I_{0}}{I_{0 R}-I_{m}} .
$$

Likewise, $R_{G T-P s}$ is the fraction of scattering due to all non-Ps scattering channels, and is given by

$$
R_{G T-P s}=\frac{I_{0}-I_{m}}{I_{0 R}-I_{m}} .
$$

By use of these ratios, the total cross sections are simply calculated from the grand total cross section as

$$
\sigma_{G T-P s}=R_{G T-P s} \sigma_{G T}
$$

and

$$
\sigma_{P s}=R_{P s} \sigma_{G T} .
$$

Consistency checks are undertaken for each set of measurements by overlapping three to five points between each adjacent experimental run. This technique provides a useful assessment of systematic uncertainty. Pressure dependency checks are carried out for each target gas to ensure we operate 
within a regime free of multiple-scattering effects. Typically the total scattering inside the gas cell was kept below $10 \%$ for these measurements.

At low energies, forward scattering becomes a significant problem and we have attempted to account for it by applying corrections based on theoretical elastic differential cross section (DCS) curves. This procedure is discussed in more detail in Sec. IV and in [6]. Results are presented with suggested corrections applied.

Sources of systematic error, such as thermal transpiration, have been estimated and, where possible, accounted for. Pressure end effects at the apertures of the scattering cell are minimized through cell design. Away from the low-energy region, the largest uncorrected source of systematic uncertainty is the zero drift in the capacitance manometer (model 690 MKS Baratron) used for pressure measurements. For $\mathrm{Ne}$ and Ar this effect is responsible for an uncertainty as large as $2 \%$. Below $10 \mathrm{eV}$, however, the uncertainty due to small-angle elastic scattering, despite correction, is expected to dominate. Typically statistical errors in the intensity measurements are less than $1 \%$ while the total absolute error is between $5 \%$ and $7 \%$.

\section{THEORETICAL APPROACH}

We present two theoretical treatments of positron scattering from Ne and Ar. One is a relativistic optical potential (ROP) method which, below the first excitation threshold, reduces to a relativistic version of the polarized orbital method. A nonrelativistic version of the polarized orbital method has been previously used to calculate the elastic cross section in neon [7] and argon [8]. The ROP calculation presented here has been used to calculate the grand total cross section below $E_{P S}$ and the cross section for the grand total minus the Ps formation channel above $E_{P s}$. The other approach that we have employed is that of the convergent close-coupling (CCC) method and is a calculation of the grand total cross section.

\section{A. The relativistic optical potential approach}

The theoretical cross sections presented here for neon and argon were determined from the solution of the relativistic Dirac-Fock scattering equations containing an $a b$ initio complex optical potential. The details of this method have been given previously in Chen et al. [9] and hence only a brief summary is given here. Basically, the optical potential is a complex potential where the real part represents the polarization of the target by the incident positron while the imaginary part of this potential, also referred to as an absorption potential, accounts for the loss of flux into inelastic channels. In the results presented here, the only inelastic channels which were included were those corresponding to direct excitation and single ionization, i.e., the Ps formation channel was not included.

As in [9], we use our polarized-orbital polarization potential [10] for the real part of our optical potential. In the case of neon, the dipole and quadrupole polarization potentials were included while for argon, with its larger multipole polarizabilities, the octopole potential was also included.
Whenever, the energy of the incident positron was such that inelastic channels (excitation and ionization) were open, the imaginary part of the optical potential was incorporated in the calculations. This absorption potential is both nonlocal and $a b$ initio, and is determined as an expansion over the inelastic channels of the target which include both the excitation of the discrete bound states as well as single ionization of the target as given by Eq. (21b) of [9]. In [9], it was shown how this absorption potential could be treated as a perturbation. However, it was subsequently found that the error introduced by this technique became larger as the incident energy of the projectile increased. Thus, we have carried out the full solution of the complex Dirac-Fock scattering equations in the work presented here.

The discrete Dirac-Fock wave functions of the target were determined using the multiconfiguration Dirac-Fock (MCDF) program of Grant et al. [11]. In the case of neon, the absorption potential included the following 15 excited states (in intermediate-coupling notation):

$\begin{array}{cccc}3 s[3 / 2]_{J=1}^{o} & 3 s[1 / 2]_{J=1}^{o} & 3 p[1 / 2]_{J=0} & 3 \bar{p}[1 / 2]_{J=0} \\ 3 p[5 / 2]_{J=2} & 3 p[3 / 2]_{J=2}^{o} & 3 \bar{p}[3 / 2]_{J=2} & 4 s[3 / 2]_{J=1}^{o} \\ 4 s[1 / 2]_{J=1}^{o} & 3 \bar{d}[1 / 2]_{J=1}^{o} & 3 d[3 / 2]_{J=1}^{o} & 3 \bar{d}[3 / 2]_{J=1}^{o} \\ 3 d[7 / 2]_{J=3}^{o} & 3 d[5 / 2]_{J=3}^{o} & 3 \bar{d}[5 / 2]_{J=3}^{o} & \end{array}$

Here $J$ represents the total angular momentum of the atom while $\bar{p}$ represents a $p$ orbital with $j=1 / 2$ and $p$ represents a $p$ orbital with $j=3 / 2$. Similarly, $d, \bar{d}$ represent $d$ orbitals with $j=5 / 2,3 / 2$ respectively. In addition, the absorption potential included the following continuum states:

$\begin{array}{llll}\epsilon s[3 / 2]_{J=1}^{o} & \epsilon s[1 / 2]_{J=1}^{o} & \epsilon p[1 / 2]_{J=0} & \epsilon \bar{p}[1 / 2]_{J=0} \\ \epsilon p[5 / 2]_{J=2} & \epsilon p[3 / 2]_{J=2} & \epsilon \bar{p}[3 / 2]_{J=2} & \epsilon \bar{d}[1 / 2]_{J=1}^{o} \\ \epsilon d[3 / 2]_{J=1}^{o} & \epsilon \bar{d}[3 / 2]_{J=1}^{o} & \epsilon d[7 / 2]_{J=3}^{o} & \epsilon d[5 / 2]_{J=3}^{o} \\ \epsilon \bar{d}[5 / 2]_{J=3}^{o} & \epsilon s[1 / 2]_{J=0} & \epsilon p[3 / 2]_{J=1}^{o} & \epsilon \bar{p}[1 / 2]_{J=1}^{o} \\ \epsilon d[5 / 2]_{J=2} & \epsilon \bar{d}[3 / 2]_{J=2} & & \end{array}$

where $\epsilon$ represents the energy of the ejected electron, originally either a bound $2 \bar{p}$ or $2 p$ electron and, above $48.5 \mathrm{eV}$, a $2 s$ electron for neon. The continuum wave functions are solutions of Eq. (50a) or (50b) of [9] with the corresponding potentials being given by Eqs. (53a) and (53b). In the case where a $2 s$ electron is ejected, the required potential is analogous to Eq. (1) of McEachran and Stauffer [12].

In the case of argon, a comparable total of 17 bound and 18 continuum states were included in the formation of the absorption potential. Here the ionization threshold for the $3 \mathrm{~s}$ electron is $29.3 \mathrm{eV}$.

Above the inelastic threshold, the scattering phase shifts are complex, i.e., $\eta_{l}^{ \pm}=\delta_{l}^{ \pm}+i \gamma_{l}^{ \pm}$, where the $+(-)$signs refer to a spin-up (spin-down) positron. The elastic and inelastic cross sections are then given by Eqs. (39) and (40) of [9], respectively. However, in the case of the pure elastic energy region, the elastic cross section is given by setting $\gamma_{l}^{ \pm}=0$ in Eq. (39).

\section{B. The convergent close-coupling approach}

A single-centered CCC method for the calculation of positron scattering from neon and argon has been developed. The single-center approach has been previously successful in the calculation of total cross sections for positron scattering 
from hydrogen [13] and helium [14,15]. The method solves the Schrödinger equation by expanding the total scattering wave function in a large set of target states and converting it to coupled Lippmann-Schwinger equations for the $T$ matrix. The target state basis is obtained by diagonalization of the target Hamiltonian in a basis constructed from Sturmian (Laguerre) one-electron functions. The resulting basis provides squareintegrable representation for the target bound states and the continuum. The single-centered CCC method does not explicitly include the positronium formation channels. However, due to completeness of the basis they are represented in the target state expansion by the positive-energy pseudostates. The total ionization cross section, which is obtained as a sum over cross sections for all positive-energy states, represents cross sections for both direct ionization by positron impact and positronium formation. This allows us to obtain a reliable estimate of total scattering for incident energies above the ionization threshold and below Ps formation, i.e., outside the Ore gap. The representation of Ps formation indirectly via single-centered expansion requires inclusion of states with high orbital angular momentum and energy [13-15].

The nonrelativistic CCC computer code has been extended to allow calculation of neon and argon target states, and the resulting scattering matrix elements. To calculate the target states we first performed self-consistent Hartree-Fock calculations for the $\mathrm{Ne}^{+} 1 s^{2} 2 s^{2} 2 p^{5}\left(\mathrm{Ar}^{+} 1 s^{2} 2 s^{2} 2 p^{5} 3 s^{3} 3 p^{5}\right)$ ground state. Next, we conducted standard configuration-interaction (CI) calculations by diagonalizing the target Hamiltonian in the basis of all possible $1 s^{2} 2 s^{2} 2 p^{5} n l\left(1 s^{2} 2 s^{2} 2 p^{5} 3 s^{3} 3 p^{5} n l\right)$ configurations. Here the active $n l$ electron is represented by the square-integrable Laguerre basis,

$\xi_{n l}(r)=\left(\frac{\lambda_{l}(n-1) !}{(2 l+1+n) !}\right)^{1 / 2}\left(\lambda_{l} r\right)^{l+1} \exp \left(-\lambda_{l} r / 2\right) L_{n-1}^{2 l+2}\left(\lambda_{l} r\right)$

where the $L_{n-1}^{2 l+2}\left(\lambda_{l} r\right)$ are the associated Laguerre polynomials, and $n$ ranges from 1 to the basis size $N_{l}$, for $0 \leqslant l \leqslant l_{\max }$. This approach allows us to model sufficiently accurately the ground and excited states of neon and argon, and is somewhat similar to the frozen-core model we have successfully utilized for $e^{-}$-He scattering [16]. In the case of neon the basis size was chosen to be $N_{l}=20-l$ and $\lambda_{l}=1.2$ for $l \leqslant l_{\max }=6$. For argon we took $N_{l}=21-l$ and $\lambda_{l}=1.8$ with $l_{\max }=6$. These lead to CCC calculations that couple 278 states for both neon and argon. The ground state ionization energy obtained is $20.60 \mathrm{eV}$ for neon and $14.97 \mathrm{eV}$ for argon. The difference from experimental ionization energies $(21.56 \mathrm{eV}$ for $\mathrm{Ne}$ and $15.76 \mathrm{eV}$ for $\mathrm{Ar}$ ) is of the same order as in the case of the helium frozen-core model. Another important check of the structure model is the static dipole polarizability. Our structure model yields $3.0 a_{0}^{3}$ for neon and $13.7 a_{0}^{3}$ for argon, which are somewhat higher than experimental values of $2.67 a_{0}^{3}$ for neon [17] and $11.08 a_{0}^{3}$ for argon [18]. These may lead to a minor overestimation of the total scattering cross section.

A number of calculations with smaller values for $N_{l}$ and $l_{\max }$ have been conducted to verify the convergence of the total scattering cross sections with respect to $N_{l}$ and $l_{\max }$. For brevity of presentation only results for the largest model are presented.

\section{RESULTS AND DISCUSSION}

In the following subsections, the present experimental and theoretical cross section results for neon and argon are presented separately. Where available, literature data are compared with the present results in the discussions for each target.

\section{A. Neon}

In Figs. 2(a) and 2(b) the present grand total cross section measurement and the present theoretical results are compared with theory and experiment, respectively, up to the Ps formation threshold $\left(E_{P S}\right)$. Below $E_{P S}$ the total cross section is, in practice, composed purely of elastic scattering (direct annihilation is negligible in this case). Corrections for low-angle scattering have been made using differential cross section curves calculated using the present ROP calculations, discussed in Sec. III. In Table I, suggested corrections are compared over a range of impact energies for positron scattering from Ne. Despite a worse energy resolution in the measurements above $4 \mathrm{eV}$, leading to a degraded angular resolution, the suggested corrections fall below $1 \%$ around $8 \mathrm{eV}$ and are within the statistical uncertainty of the present measurements.

Below $E_{P s}$ the cross section is dominated by a deep Ramsauer-Townsend (RT) minimum. The minimum is found to be centered between 0.6 and $0.7 \mathrm{eV}$ in the present measurement, with the minimum value of $\sigma_{G T}$ found to be approximately $0.14 \AA^{2}$. The ROP and CCC theories both find the minimum at about $0.7 \mathrm{eV}$, with magnitudes of approximately 0.12 and $0.13 \AA^{2}$, respectively. The total cross section climbs sharply out of the minima, and by $12 \mathrm{eV}$ has almost plateaued, reaching a maximum value of approximately $0.9 \AA^{2}$ at $14 \mathrm{eV}$ in the present measurement. This magnitude is in close agreement with that found in the ROP calculation. However the present CCC result is approximately $10 \%$ higher at around $1 \AA^{2}$.

In Fig. 2(a) the present measurement and theoretical predictions of the grand total cross section $\left(\sigma_{G T}\right)$ are compared with selected theoretical calculations for impact energies up to $E_{P S}$. Each of the theoretical predictions produces cross sections that are broadly consistent with the present results.

Prior to the work presented here, the two calculations in best agreement are those of McEachran et al. [7] and Dzuba et al. [22], which are approximately equidistant from the present result over most of the range. The many-body

TABLE I. Corrections for forward-angle scattering in neon. Up to $4 \mathrm{eV}, \Delta V=0.065 \mathrm{~V}$; above that, $\Delta V=0.15 \mathrm{~V}$. See text for details.

\begin{tabular}{lcc}
\hline \hline Energy $(\mathrm{eV})$ & $\theta_{\min }(\mathrm{deg})$ & TCS correction $(\%)$ \\
\hline 0.3 & 27.7 & 24.0 \\
0.5 & 21.1 & 23.0 \\
1.0 & 14.8 & 13.9 \\
2.0 & 10.4 & 4.6 \\
4.0 & 7.3 & 1.5 \\
5.0 & 10.0 & 2.3 \\
6.0 & 9.1 & 1.8 \\
8.0 & 7.9 & 1.1 \\
10.0 & 7.0 & 0.8 \\
12.0 & 6.4 & 0.6 \\
\hline \hline
\end{tabular}




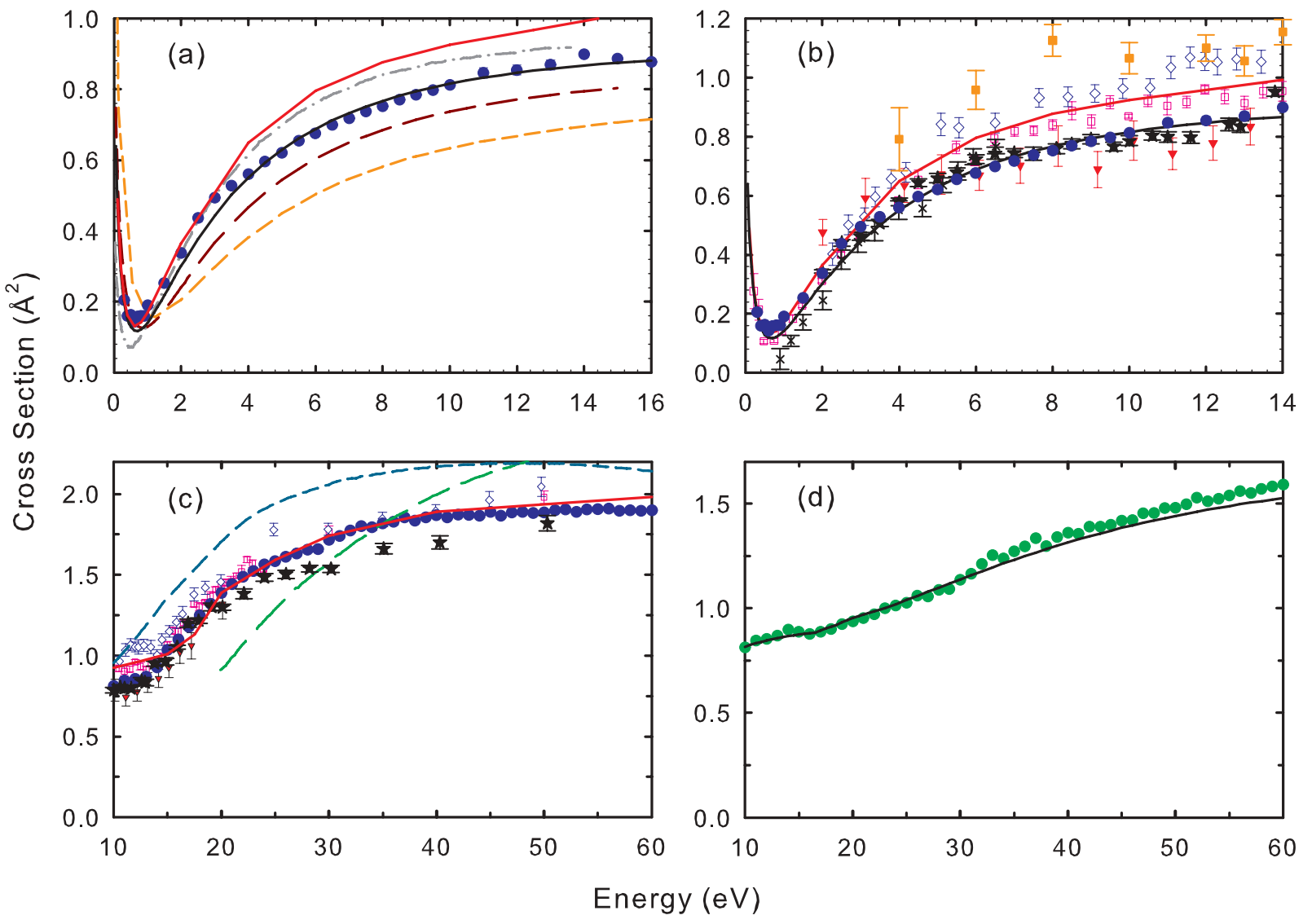

FIG. 2. (Color online) Cross sections for positron scattering from Ne. (a) Comparison of the present $\sigma_{G T}$ below $E_{P s}$ with theoretical models. (b) Comparison of the present $\sigma_{G T}$ below $E_{P s}$ with other experimental results. (c) The present $\sigma_{G T}$ above $E_{P S}$ compared with other experimental and theoretical results. (d) The present $\sigma_{G T-P s}$ above $E_{P s}$ compared with the present result of the ROP approach. $\bullet$, present $\sigma_{G T}$; -, present $\sigma_{G T-P s}$; -, present polarized orbital result; - , present CCC result; --, Coleman et al. [19]; - -, McEachran et al. [7]; - - - -, Nakanishi and Schrader [20]; --, Baluja and Jain [21]; -.-, Dzuba et al. [22]; $\mathbf{\nabla}$, Canter et al. [23]; $\square$, Jaduszliwer and Paul [24]; $\star$, Coleman et al. [19]; $\square$, Stein et al. [25]; $\times$ Sinapius et al. [26]; $\diamond$, Charlton et al. [27].

perturbation theory of Dzuba et al. [22] predicts a position of the RT minimum that is in good agreement with that found in the present experimental result, although the magnitude of the cross section at the position of the minimum is about half that found experimentally. Above $5 \mathrm{eV}$ the calculated cross section lies above the present measurement, on average by about $10 \%$, but appears to plateau to a magnitude close to that presently found near $E_{P s}$. Similarly, the present CCC result reproduces the cross section well at impact energies below $3 \mathrm{eV}$, but continues to diverge from the present result up to $E_{P s}$. Conversely, the polarized orbital calculation of McEachran et al. [7] lies approximately $10 \%$ below the present result above $2 \mathrm{eV}$ and predicts a minimum centered at about $1 \mathrm{eV}$, although again of lower magnitude than that presently measured. The present ROP calculation, below $E_{P S}$, reduces to a relativistic form of the polarized orbital approach and reproduces the present measured cross section more closely than the other theoretical predictions compared here. The work of Nakanishi and Schrader [20] employed a simplified polarization model that cut off the real behavior of the polarization potential at the boundary of the atom, employing a constant value inside. The relatively poor agreement in both magnitude and shape demonstrates the importance of properly accounting for polarization at low energies, even in the smaller noble gases where polarization is relatively weak.

In Fig. 2(b) we compare the present experimental result and theories with previous experimental data. The earliest measurements, those of Canter et al. [23], compare favorably over the measured range with the present experimental data, although they appear to underestimate the cross section by about $5 \%$ between 8 and $14 \mathrm{eV}$. At the lowest measured energy, around $2 \mathrm{eV}$, their result lies well above the present data. The measurements of Coleman et al. [28] and Sinapius et al. [26] are in similarly good agreement with the present data, and by extension the present ROP calculation, though the measurements of Sinapius et al. lie below the present measurements from $2 \mathrm{eV}$ and below. Conversely, the data of Stein et al. [25] compare well below $5 \mathrm{eV}$, predicting a minimum that is in close agreement in energy to that presently found, but that lies about $20 \%$ below the present in magnitude. This result is likely to be due to the better energy resolution, and thus angular discrimination, of the present measurements. In the experiment of Stein et al. the maximum angle at which elastically scattered positrons are indistinguishable from the unscattered beam, in the region of the minimum, is estimated to be about $13^{\circ}$. Comparison of the total cross section calculated from the 
ROP DCS with a reduced total cross section integrated over $13^{\circ}-180^{\circ}$ suggests that their measured cross section would be approximately $12 \%$ lower than the true total cross section. This accounts for a substantial fraction of the difference between their results and the present measurements. Accounting for other possible systematic and statistical uncertainties places the two measurements in reasonable accord. At higher energies it is more difficult to account for the difference in the magnitude of the cross section, where the present results are typically about $10 \%$ larger than those of [25], although the difference is not substantially greater than the stated errors between the measurements. The present $\mathrm{CCC}$ result lies close to the results of Stein et al. away from the minimum. The later results of Charlton et al. [27] start around $2 \mathrm{eV}$ and are initially in good agreement with the present measurement. Above $4 \mathrm{eV}$, however, their cross section lies substantially above the present measurements, by as much as $30 \%$, although typically the difference is closer to $15 \%-20 \%$, which makes it in reasonable agreement with the present $\mathrm{CCC}$ result. The data of Jaduszliwer and Paul [24] lie higher again over the range studied, between $10 \%$ and $15 \%$ above the CCC curve and as much as $25 \%-30 \%$ above the present measurement and ROP calculation.

In Fig. 2(c) the total cross section above $E_{P s}$ is shown alongside available experimental results and theory. The grand total cross section rises sharply from the onset of Ps formation at $14.76 \mathrm{eV}$ due to the new scattering channel, and continues rising as electronic excitations and ionization become energetically available. The grand total cross section appears to plateau around $60 \mathrm{eV}$ with a maximum value of approximately $1.9 \AA^{2}$ found in the present measurements. The present CCC result is in close agreement with the present measurement at energies above the ionization threshold, differing from the present data by less than $5 \%$ up to $60 \mathrm{eV}$. Between $E_{P s}$ and the ionization threshold the theory is unable to account for Ps formation and subsequently falls below the present measurement. Baluja and Jain [21] employed an optical potential model which attempted to describe scattering at intermediate energies. The range studied extends from relatively low energies, where the present results lie, up to higher impact energies, where the Born approximation applies. The peak in the grand total cross section, presently found around $50-60 \mathrm{eV}$, is found closer to $75 \mathrm{eV}$ in their prediction, and the magnitude of the cross section significantly exceeds that presently measured. Below $35 \mathrm{eV}$, where their curve intersects the present data, the difference is as much as $50 \%$. The application of the sum rule by Coleman et al. [19] was interpolated from experimental results, taken in a different apparatus from the experimental data of Coleman et al. [28] compared here. The shape of the theoretical curve is similar to the observed cross section, but the magnitude is significantly above the present result, as well as the later measurements of Coleman et al.

The experimental data of Coleman et al. are in generally good agreement with the present result up to $20 \mathrm{eV}$, but the two results diverge at higher energies, with the result of [28] lying approximately $10 \%$ below the present measurement around $30 \mathrm{eV}$ before converging back into agreement around $50 \mathrm{eV}$. The results of Canter et al. [23], although lower, remain in reasonable agreement with the present data up to $20 \mathrm{eV}$ where their data end. The measurements of both Stein et al. [25] (which incorporate the data of Kauppila et al. [29] above $30 \mathrm{eV}$ ) and those of Charlton et al. [27] both tend toward the present data around $20 \mathrm{eV}$ and remain in reasonable agreement at higher energies.

Above $E_{P s}$ the present ROP calculation is unable to account for the Ps formation cross section, but can account for electronic excitation and ionization through an absorption potential. This part of the calculation can be compared to the measured GT - Ps cross section and is shown in Fig. 2(d). Agreement with the measured $\sigma_{G T-P s}$ is excellent over the region investigated, and suggests the possibility that the Ps formation channel has little interaction with the other scattering channels at higher energies above the Ps threshold, although comparison with partial total cross sections would clearly be more illuminating.

Centred about $E_{P s}$, below the first excitation threshold at $16.62 \mathrm{eV}$, a weak cusp is visible in the measurements of $\sigma_{G T-P s}$. In this range, known as the Ore gap, measurements of $\sigma_{G T-P S}$ correspond to the total elastic cross section. The cusp is thought to arise from strong channel coupling between Ps formation and elastic scattering, and has been studied in more detail in a previous publication [30].

In Fig. 3 we compare the present measurement of the total Ps formation cross section with the measurements of Laricchia et al. [31] and Marler et al. [32] as well as with the theoretical curves of McAlinden et al. [33] and Gilmore et al. [34]. From threshold up to about $25 \mathrm{eV}$ the three measurements are in generally good agreement. Above $25 \mathrm{eV}$ the prior measurements plateau at approximately $0.45 \AA^{2}$ before turning over and diverging slightly from one another above $35 \mathrm{eV}$ impact energy. The present measurements instead continue to climb gradually to a maximum value of approximately $0.53 \AA^{2}$ at $30 \mathrm{eV}$. Above $40 \mathrm{eV}$ the present result falls below the measurements of Laricchia et al., while remaining slightly above the results of Marler et al. Disagreement with the results of Marler et al. is perhaps explained by the application of the single-scattering approximation in deriving $\sigma_{P S}$ in their work.

The present results for $\sigma_{P s}$ are determined by substituting Eqs. (2) and (3) into Eq. (6) as discussed in Sec. II. The

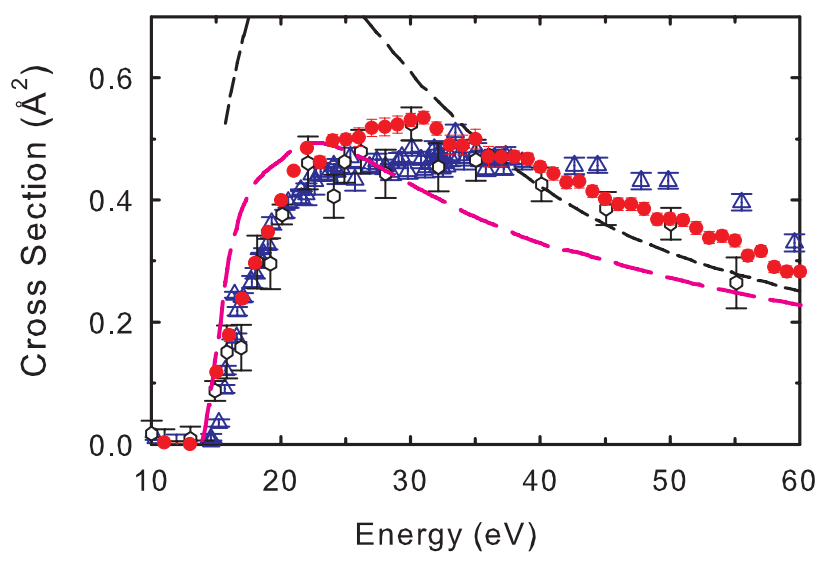

FIG. 3. (Color online) Comparison of the present positronium formation cross section $\left(\sigma_{P S}\right)$ for positron scattering from $\mathrm{Ne}$ with other experimental and theoretical results. - present $\sigma_{P S}$ result; - - - , McAlinden and Walters [33]; _ _, Gilmore et al. [34]; $\triangle$, Laricchia et al. [31]; ○, Marler et al. [32]. 
TABLE II. Corrections for forward-angle scattering in argon. $\Delta V=0.065 \mathrm{~V}$. TCS indicates the total cross section.

\begin{tabular}{lcc}
\hline \hline Energy $(\mathrm{eV})$ & $\theta_{\min }(\mathrm{deg})$ & TCS correction $(\%)$ \\
\hline 0.3 & 27.7 & 16.3 \\
0.5 & 21.1 & 13.4 \\
1.0 & 14.8 & 12.5 \\
2.0 & 10.4 & 9.4 \\
4.0 & 7.3 & 5.1 \\
6.0 & 6.0 & 3.5 \\
8.5 & 5.0 & 2.4 \\
\hline \hline
\end{tabular}

single-scattering approximation is given below:

$$
\sigma_{P s}=\frac{1}{n_{m} l} \frac{\Delta I_{P s}}{I_{0 R}},
$$

where $\Delta I_{P s}$ is the flux lost to Ps formation, and all other variables are as described previously.

If we apply the single-scattering approximation to the present data we find the cross section value at the peak drops into relatively good agreement with the measurements of Marler et al. and, up to approximately $40 \mathrm{eV}$, is in good agreement with the data of Laricchia et al.

Comparison with theory is limited to a few approximate methods. The work of McAlinden and Walters [33] applies a truncated, coupled-static approximation. Above $35 \mathrm{eV}$ the calculated cross section is in qualitative agreement with the present measurement, but the peak position is found almost $10 \mathrm{eV}$ below the peak value of the present measurements and is approximately $50 \%$ greater in magnitude. The later work of Gilmore et al. [34], using the distorted wave Born approximation (DWBA), is somewhat similar in shape to the curve of McAlinden and Walters and, in their original work, was scaled by a factor of 0.57 in order to bring the magnitude into agreement with the results of Laricchia $e t a l$.

\section{B. Argon}

In Table II the corrections applied to the present results to account for forward scattering are given for a few selected energies. Note that in contrast to the corrections applied to the $\mathrm{Ne}$ results, the magnitude of the corrections is initially smaller, at energies below $2 \mathrm{eV}$, despite the use of an identical experimental arrangement, and thus an identical angular discrimination. From impact energies of $2 \mathrm{eV}$ and up the $\mathrm{Ne}$ corrections rapidly diminish to an insignificant level, falling below $2 \%$ at $6 \mathrm{eV}$ for example, despite a worsening in the angular discrimination at energies above $4 \mathrm{eV}$. In $\mathrm{Ar}$ the angular discrimination employed is better than that in $\mathrm{Ne}$ above $4 \mathrm{eV}$, yet the suggested correction near the Ps formation threshold remains slightly above $2 \%$. This is due to the more forward-peaked differential elastic scattering cross section in argon relative to neon in the present ROP calculations, which are used in determining the corrections. As we see in Fig. 4(a), one may initially think that there is reason to be cautious about the applied corrections in the case of Ar, as agreement of the present experimental result with the present ROP calculation is relatively poor. However, we have demonstrated in [6] that the corrections that result are relatively insensitive to the theoretical model that is used.
In Fig. 4(a) the present measurement and theoretical calculations of $\sigma_{G T}$ are compared with other selected theoretical predictions. The work of Montgomery and LaBahn [35] is the earliest result compared here and employs a polarized-orbital method, similar to that used in the present ROP calculation below $E_{P s}$ as well as in the calculations of McEachran et al. [8] and Nakanishi and Schrader [20]. The differences in these similar calculations highlight the importance of properly handling the effect of polarization. Montgomery and LaBahn treat the polarization effect only at long range and as a result their calculation significantly underestimates the observed magnitude, most notably at low energies. Similarly, in Nakanishi and Schrader's work, the polarization was treated properly at long distance, but is truncated inside the effective radius of the atom through use of a cutoff function. McEachran et al. numerically solved the effect of polarization inside the atomic charge cloud and included higher-order polarization terms in their calculation. This approach is essentially identical to that of the present ROP calculation; however, the present calculation includes the polarization terms only up to the octopole polarizability for Ar. Jain [36] applied a parameterfree model that treated the polarization inside the atom analogously to the behavior of a positron in a free-electron cloud, and then matched this behavior to the correct asymptotic behavior at long distance. With the exception of Nakanishi and Schrader's calculation, each of the polarized-orbital approaches tends to converge to a common value near $E_{P s}$ of about $3 \AA^{2}$, well below the present measurement, which finds a value closer to $4 \AA^{2}$. The many-body calculations of Dzuba et al. [22] find better agreement with the present result above $2 \mathrm{eV}$ than the various polarized-orbital approximations which are considered. They also demonstrated in their original work a significant contribution to the total cross section, even at low energy, from virtual Ps formation. Around $1 \mathrm{eV}$, however, there is a minimum in their predicted cross section that is not seen in the present experimental data. The present CCC result appears to provide the best agreement with the experimental data across the energy range below $E_{P s}$, although it appears to overestimate the cross section below $4 \mathrm{eV}$.

In Fig. 4(b) we compare the present grand total cross section with other experimental results below $E_{P s}$. Between $3 \mathrm{eV}$ and $E_{P s}$ there is a general consensus among the measurements of Kauppila et al. [38], Charlton et al. [27], Sinapius et al. [26], and the recent results of Karwasz et al. [39], all finding relatively constant values of $\sigma_{G T}$ that lie around $3 \AA^{2}$. This value is in relatively good agreement with the magnitude predicted by most of the theoretical calculations compared in Fig. 4(a), including the present ROP calculation, which is in broad agreement with those prior experimental measurements over most of the energy range. Across this same region, between 3 and $9 \mathrm{eV}$, the present measurements, as well as those of Canter et al. [23], Jaduszliwer and Paul [24], and to a lesser extent the single measurement of Coleman et al. [40] at $5 \mathrm{eV}$, find a magnitude between 3.7 and $4 \AA^{2}$.

Below $3 \mathrm{eV}$ the results of Canter et al. fall below the present measurements, finding agreement with the results of Kauppila et al. at $2 \mathrm{eV}$. Similarly, the data of Coleman et al. do not find evidence of the sharply rising cross section seen in the data of Kauppila et al., Karwasz et al., and the present results below $1.5 \mathrm{eV}$, predicting a value less than one-half that presently 

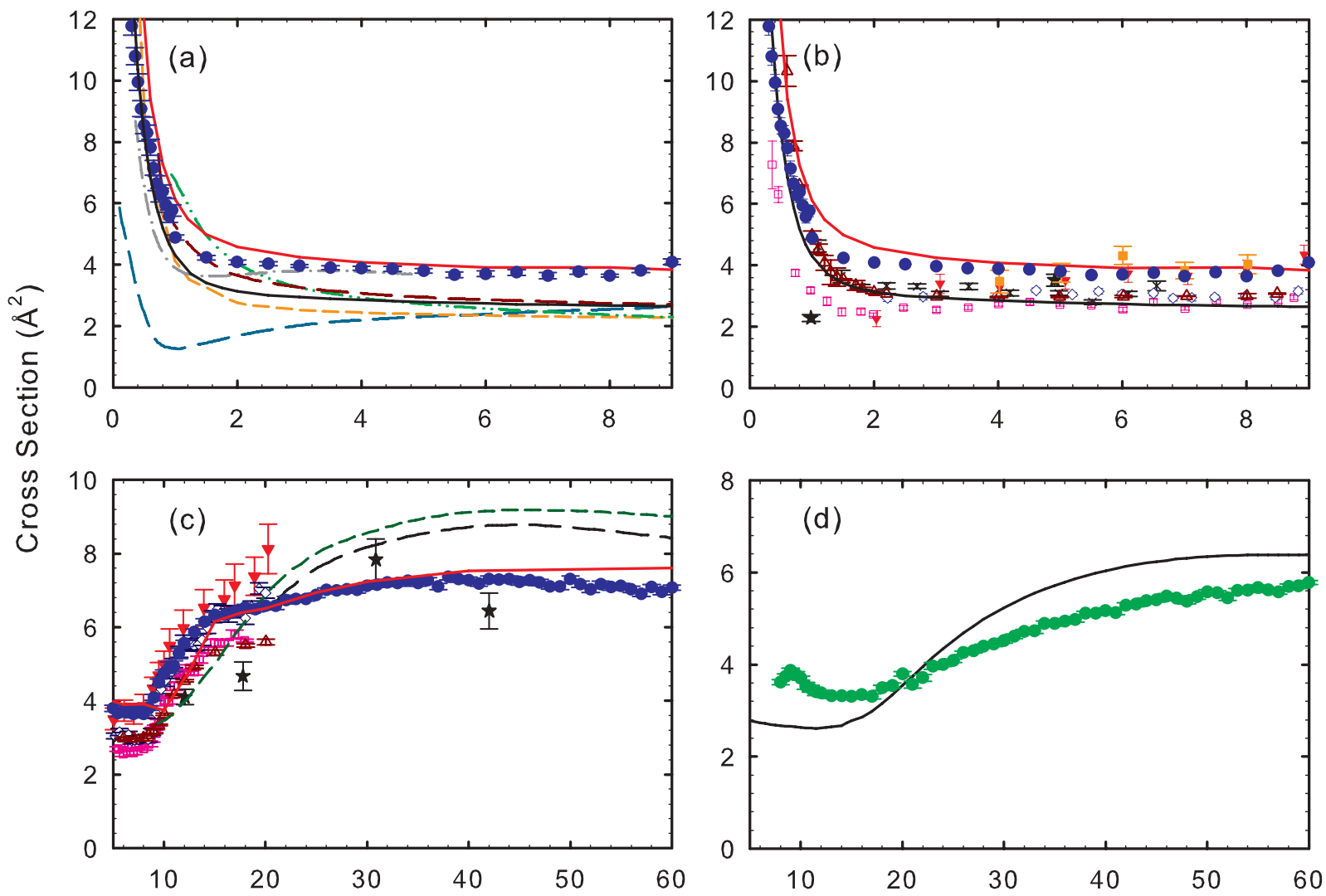

Energy (eV)

FIG. 4. (Color online) Cross sections for positron scattering from Ar. (a) Present $\sigma_{G T}$ below $E_{P s}$ compared with theory. (b) Present $\sigma_{G T}$ below $E_{P s}$ compared with experiment. (c) Present $\sigma_{G T}$ compared with theory and experiment above $E_{P s}$. (d) Present $\sigma_{G T-P s}$ compared with the present ROP calculation above $E_{P s} . \bullet$, present $\sigma_{G T}$; ๑, present $\sigma_{G T-P s} ;-$, present ROP result; —, present CCC result; - -, Montgomery and LaBahn [35]; — —, McEachran et al. [8]; - - - , Nakanishi and Schrader [20]; - - -, Jain [36]; - - -, Nahar and Wadehra [37]; —- Baluja and Jain [21]; -.-, Dzuba et al. [22]; $\mathbf{\nabla}$, Canter et al. [23]; $\square$, Jaduszliwer and Paul [24]; $\square$, Kauppila et al. [38]; $\star$, Coleman et al. [19]; $\times$, Sinapius et al. [26]; $\diamond$, Charlton et al. [27]; $\triangle$, Karwasz et al. [39].

observed. The results of Karwasz et al. are in reasonable agreement with the present results below $1.5 \mathrm{eV}$, although they rise notably above at the lowest energies, lying in good agreement with the present CCC calculation. The poor agreement among experimental results has been explored previously [1]. However, a thorough examination of sources of systematic error was insufficient to explain the disparity in results, except for the uncertainty in the magnitude of corrections required for the problem of forward angle scattering. Preliminary measurements with the present apparatus [6,41], performed with a degraded angular resolution, found a cross section that was in relatively good agreement with the result of Kauppila et al., including observation of an apparent minimum in the cross section around $1.5 \mathrm{eV}$. Corrections applied to the preliminary results were clearly insufficient in light of the present measurements, as the corrected results fell below the present data by approximately $30 \%$, significantly below even the present results prior to correction. We now understand the reason for this due to our better understanding of the angular discrimination problems. This result, as well as the apparent emergence of the Ramsauer-Townsend minimum observed by Kauppila et al., has been discussed in more detail in a recent study [6].
Previous experimental and theoretical results are compared with the present results for impact energies above $E_{P s}$ in Fig. 4(c). The experimental results again fall broadly into two groups. The results of Kauppila et al., Karwasz et al., and Coleman et al. all remain below the present result, lying about $1 \AA^{2}$ lower up to $20 \mathrm{eV}$ impact energy. In this range the data of Kauppila et al. and Karwasz et al. are in good agreement with one another. Above $20 \mathrm{eV}$ the measurements of Coleman et al. are found to be in reasonable agreement with the present measurement, although the data are sparse. The results of Canter et al. remain in good agreement with the present result up to $15 \mathrm{eV}$, diverging slightly above the present result at higher energies, ending about $15 \%$ higher at $20 \mathrm{eV}$. The result of Charlton et al. rises up sharply after the Ps formation threshold and lies in good agreement with the present result up to $20 \mathrm{eV}$.

Theoretical calculations for the energy range from $E_{P s}$ up to $60 \mathrm{eV}$, the limit of the present measurements, are again quite limited, as in neon. The work of Nahar and Wadehra [37] applied the relativistic Dirac equation, using a sum of model potentials to describe the interaction of the positron and target atom. In their work Nahar and Wadehra found that for different model potentials they were able to obtain an integrated cross 
section in reasonable agreement with experiment, but the differences in partial cross sections could be substantial. We find that the present result is in poor agreement with this calculation, although the predicted cross section appears to find reasonable agreement at low energy with the measurements of Kauppila et al. and Karwasz et al. The smooth rise and subsequent fall observed in their calculation shows no evidence of the sharp flattening of the cross section which is observed in the present result around $15 \mathrm{eV}$. The complex optical potential calculation of Baluja and Jain [21] follows a similar trend to that in [27], although the predicted cross section is slightly lower than that of Nahar and Wadehra. It lies closer to the present result, but rises to a maximum which is about $20 \%$ larger than that found presently at approximately $45 \mathrm{eV}$. As in $\mathrm{Ne}$, the present $\mathrm{CCC}$ result provides the best theoretical prediction of $\sigma_{G T}$ in the intermediate-energy range studied. The region between $E_{P s}$ and the ionization threshold is again a region of poor agreement, and similarly the predicted cross section diverges from the present measurement at the highest impact energies, lying approximately $6 \%$ above the present measurement at $60 \mathrm{eV}$.

In Fig. 4(d) the present ROP result is compared with the present $\sigma_{G T-P s}$ measurements. Unlike the comparison in $\mathrm{Ne}$, there is relatively poor agreement seen here. The predicted cross section at $E_{P S}$ begins approximately $25 \%$ below the present measurements and, due to the lack of inclusion of the Ps formation channel, fails to predict the observed cusp, centered about $E_{P S}$ (see [30] for more details). Above $15 \mathrm{eV}$ the calculated cross section turns upward and rises rapidly compared with the measurement, approaching a maximum value of about $7 \AA^{2}$ at $60 \mathrm{eV}$, approximately the same magnitude as the maximum $\sigma_{G T}$ which is presently measured.

The present measurements of $\sigma_{P s}$ are compared with other recent experimental results and theoretical predictions in Fig. 5. The present measurement rises sharply, and approximately linearly, from threshold with increasing energy, with a slope of about $0.63 \AA^{2}$ per $\mathrm{eV}$. The cross section peaks at $17 \mathrm{eV}$, reaching a maximum value of $3 \AA^{2}$, after which it diminishes rather monotonically between 25 and $60 \mathrm{eV}$.

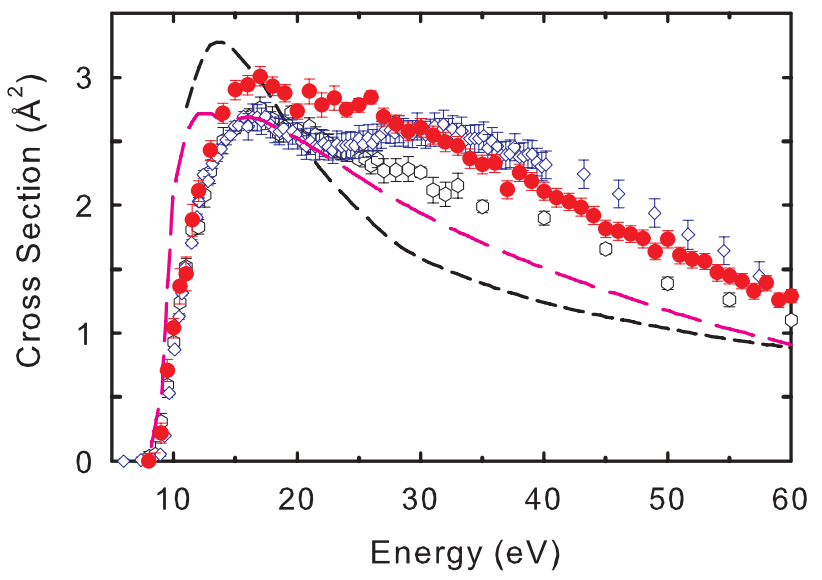

FIG. 5. (Color online) Comparison of the present positronium formation cross section $\left(\sigma_{P S}\right)$ for positron scattering from Ar with available theory and experiment. $\bullet$, present $\sigma_{P_{s}}$; - - - -, McAlinden and Walters [33]; ——, Gilmore et al. [34]; $\diamond$, Laricchia et al. [31]; $\checkmark$, Marler et al. [32].
As in $\mathrm{Ne}$, the comparison with measurements of $\sigma_{P s}$ has been limited to the work of Laricchia et al. [31] and Marler et al. [32]. In their measurements Laricchia et al. observe a second peak in the cross section, occurring around 30-32 eV, that is not seen in the present data. The present result finds agreement in shape with the measurements of Marler et al., but again differs in absolute magnitude. As discussed in the analysis of the $\mathrm{Ne}$ results, this difference is understood to arise primarily from the use of the singlescattering approximation in the work of Marler et al., whereas the present results are derived using the Beer-Lambert law. The larger disagreement observed in Ar, about $9 \%$ at the peak, could be further attributed to the larger systematic uncertainty in both experimental results due to the lower gas pressures required to maintain the total scattering fraction to around $10 \%$ of the incident beam. The present experiment employs a high-accuracy baratron gauge, and due to the shorter scattering cell, is operated at higher target pressures, reducing the uncertainty in the pressure measurement, which may explain some of the discrepancy.

Comparison with theoretical predictions is again limited to the coupled static approximation of McAlinden and Walters [33] and the DWBA calculations of Gilmore et al. [34]. The data of Gilmore et al. have been scaled by a factor of 2 to find agreement with the peak magnitude of the experimental results of Laricchia et al., and are intended to provide a theoretical basis for the observed double-peak feature. The prediction of McAlinden and Walters again provides the best comparison in terms of cross section magnitude, although comparison with the energy dependence is again poor. Their predicted cross section rises sharply above threshold, peaking at a lower energy and to a higher magnitude than the compared experimental results. The peak in the cross section of [33] lies about $3 \mathrm{eV}$ below that found in the present data, and is approximately $8 \%$ larger, reaching a maximum value around $3.25 \AA^{2}$. At scattering energies above $14 \mathrm{eV}$ the calculation drops away more rapidly than in any of the measurements. The calculated cross section reaches a value at $60 \mathrm{eV}$ that is approximately $70 \%$ of the present value.

\section{CONCLUDING REMARKS}

We have presented measurements of $\sigma_{G T}, \sigma_{G T-P s}$, and $\sigma_{P s}$ cross sections (see Table III), alongside calculations using the relativistic optical potential approach and using the convergent close-coupling approach, for positron scattering from $\mathrm{Ne}$ and Ar up to $60 \mathrm{eV}$ impact energy.

In $\mathrm{Ne}$ the present $\sigma_{G T}$ result is generally consistent with other measurements, with the largest disparity among measurements being seen below $E_{P s}$, while measurements tend to converge at higher energies. Comparison of the present $\sigma_{G T}$ below $E_{P s}$ with the present ROP result is excellent, while above $E_{P s}$, the comparison of the ROP result with the $\sigma_{G T-P s}$ measurement is equally encouraging. Comparison of the present $\sigma_{G T}$ with the results of the CCC theory is good throughout most of the energy range explored, although below $E_{P S}$ the CCC result is typically in better agreement with the results of Kauppila et al. than the present measurements. 
TABLE III. Tabulated measurements of the grand total, $\sigma_{G T}$, the grand total without Ps formation, $\sigma_{G T-P s}$, and the Ps formation cross section, $\sigma_{P s}$, for both $\mathrm{Ne}$ and Ar. Corrections for small-angle scattering have been applied to the elastic portion of the cross sections up to $13 \mathrm{eV}$ in $\mathrm{Ne}$ and $15 \mathrm{eV}$ in Ar, as outlined in Sec. IV. Cross sections and statistical errors are given in units of $10^{-16} \mathrm{~cm}^{2}$. Scattering energy $E$ is given in $\mathrm{eV}$.

\begin{tabular}{|c|c|c|c|c|c|c|c|c|c|c|c|c|}
\hline \multirow[b]{2}{*}{$E$} & \multicolumn{6}{|c|}{ Neon } & \multicolumn{6}{|c|}{ Argon } \\
\hline & $\sigma_{G T}$ & Error & $\sigma_{G T-P s}$ & Error & $\sigma_{P s}$ & Error & $\sigma_{G T}$ & Error & $\sigma_{G T-P s}$ & Error & $\sigma_{P s}$ & Error \\
\hline 0.300 & 0.204 & 0.008 & & & & & 11.774 & 0.290 & & & & \\
\hline 0.350 & & & & & & & 10.788 & 0.273 & & & & \\
\hline 0.400 & 0.160 & 0.008 & & & & & 9.950 & 0.268 & & & & \\
\hline 0.450 & & & & & & & 9.081 & 0.258 & & & & \\
\hline 0.500 & 0.162 & 0.008 & & & & & 8.538 & 0.266 & & & & \\
\hline 0.550 & & & & & & & 8.291 & 0.257 & & & & \\
\hline 0.600 & 0.142 & 0.007 & & & & & 7.820 & 0.261 & & & & \\
\hline 0.650 & & & & & & & 7.153 & 0.242 & & & & \\
\hline 0.700 & 0.158 & 0.007 & & & & & 6.653 & 0.241 & & & & \\
\hline 0.750 & & & & & & & 6.265 & 0.228 & & & & \\
\hline 0.800 & 0.160 & 0.007 & & & & & 6.392 & 0.210 & & & & \\
\hline 0.850 & & & & & & & 5.931 & 0.219 & & & & \\
\hline 0.900 & 0.160 & 0.007 & & & & & 5.575 & 0.201 & & & & \\
\hline 0.950 & & & & & & & 5.768 & 0.189 & & & & \\
\hline 1.000 & 0.190 & 0.006 & & & & & 4.887 & 0.082 & & & & \\
\hline 1.500 & 0.253 & 0.010 & & & & & 4.229 & 0.077 & & & & \\
\hline 2.000 & 0.337 & 0.009 & & & & & 4.079 & 0.074 & & & & \\
\hline 2.500 & 0.437 & 0.010 & & & & & 4.029 & 0.067 & & & & \\
\hline 3.000 & 0.494 & 0.009 & & & & & 3.962 & 0.046 & & & & \\
\hline 3.500 & 0.527 & 0.009 & & & & & 3.896 & 0.064 & & & & \\
\hline 4.000 & 0.560 & 0.003 & & & & & 3.886 & 0.064 & & & & \\
\hline 4.500 & 0.596 & 0.003 & & & & & 3.861 & 0.081 & & & & \\
\hline 5.000 & 0.620 & 0.003 & & & & & 3.796 & 0.080 & & & & \\
\hline 5.500 & 0.655 & 0.003 & & & & & 3.667 & 0.090 & & & & \\
\hline 6.000 & 0.675 & 0.002 & & & & & 3.699 & 0.090 & & & & \\
\hline 6.500 & 0.699 & 0.002 & & & & & 3.746 & 0.092 & & & & \\
\hline 7.000 & 0.717 & 0.002 & & & & & 3.641 & 0.094 & & & & \\
\hline 7.500 & 0.737 & 0.003 & & & & & 3.777 & 0.097 & & & & \\
\hline 8.000 & 0.752 & 0.003 & & & & & 3.643 & 0.045 & 3.618 & 0.051 & 0.000 & 0.000 \\
\hline 8.500 & 0.770 & 0.003 & & & & & 3.811 & 0.064 & 3.743 & 0.053 & -0.006 & 0.072 \\
\hline 9.000 & 0.784 & 0.003 & & & & & 4.089 & 0.089 & 3.871 & 0.051 & 0.218 & 0.078 \\
\hline 9.500 & 0.797 & 0.003 & & & & & 4.497 & 0.094 & 3.789 & 0.052 & 0.708 & 0.088 \\
\hline 10.000 & 0.813 & 0.003 & 0.813 & 0.003 & -0.007 & 0.003 & 4.751 & 0.084 & 3.708 & 0.045 & 1.040 & 0.076 \\
\hline 10.500 & & & & & & & 4.898 & 0.153 & 3.533 & 0.076 & 1.365 & 0.140 \\
\hline 11.000 & 0.846 & 0.015 & 0.846 & 0.015 & 0.003 & 0.007 & 4.939 & 0.143 & 3.475 & 0.072 & 1.464 & 0.132 \\
\hline 11.500 & & & & & & & 5.288 & 0.143 & 3.402 & 0.075 & 1.885 & 0.125 \\
\hline 12.000 & 0.854 & 0.014 & 0.854 & 0.014 & -0.006 & 0.007 & 5.565 & 0.087 & 3.381 & 0.054 & 2.112 & 0.081 \\
\hline 13.000 & 0.868 & 0.014 & 0.868 & 0.014 & 0.000 & 0.007 & 5.867 & 0.087 & 3.319 & 0.052 & 2.428 & 0.079 \\
\hline 14.000 & 0.926 & 0.008 & 0.898 & 0.007 & -0.013 & 0.006 & 6.143 & 0.083 & 3.317 & 0.053 & 2.716 & 0.079 \\
\hline 15.000 & 1.035 & 0.008 & 0.887 & 0.007 & 0.118 & 0.005 & 6.326 & 0.080 & 3.306 & 0.052 & 2.901 & 0.076 \\
\hline 16.000 & 1.099 & 0.010 & 0.876 & 0.008 & 0.178 & 0.009 & 6.332 & 0.083 & 3.341 & 0.052 & 2.938 & 0.077 \\
\hline 17.000 & 1.178 & 0.008 & 0.887 & 0.007 & 0.238 & 0.008 & 6.422 & 0.081 & 3.310 & 0.051 & 3.005 & 0.076 \\
\hline 18.000 & 1.249 & 0.008 & 0.900 & 0.007 & 0.296 & 0.007 & 6.536 & 0.079 & 3.491 & 0.052 & 2.930 & 0.071 \\
\hline 19.000 & 1.319 & 0.008 & 0.924 & 0.007 & 0.347 & 0.008 & 6.490 & 0.074 & 3.543 & 0.053 & 2.874 & 0.068 \\
\hline 20.000 & 1.389 & 0.006 & 0.936 & 0.006 & 0.400 & 0.006 & 6.583 & 0.069 & 3.796 & 0.053 & 2.733 & 0.059 \\
\hline 21.000 & 1.442 & 0.006 & 0.952 & 0.006 & 0.447 & 0.006 & 6.573 & 0.098 & 3.563 & 0.071 & 2.890 & 0.098 \\
\hline 22.000 & 1.487 & 0.006 & 0.971 & 0.006 & 0.485 & 0.006 & 6.730 & 0.098 & 3.712 & 0.074 & 2.783 & 0.096 \\
\hline 23.000 & 1.521 & 0.010 & 1.000 & 0.008 & 0.462 & 0.010 & 6.767 & 0.095 & 3.966 & 0.075 & 2.837 & 0.095 \\
\hline 24.000 & 1.563 & 0.010 & 1.013 & 0.008 & 0.497 & 0.010 & 6.767 & 0.062 & 3.995 & 0.044 & 2.748 & 0.056 \\
\hline 25.000 & 1.584 & 0.010 & 1.025 & 0.008 & 0.499 & 0.009 & 6.868 & 0.061 & 4.082 & 0.041 & 2.782 & 0.056 \\
\hline 26.000 & 1.612 & 0.015 & 1.059 & 0.012 & 0.503 & 0.015 & 7.007 & 0.061 & 4.256 & 0.042 & 2.844 & 0.052 \\
\hline 27.000 & 1.631 & 0.015 & 1.056 & 0.012 & 0.518 & 0.014 & 6.989 & 0.082 & 4.300 & 0.051 & 2.689 & 0.069 \\
\hline 28.000 & 1.656 & 0.014 & 1.087 & 0.012 & 0.519 & 0.015 & 7.031 & 0.085 & 4.396 & 0.047 & 2.635 & 0.072 \\
\hline 29.000 & 1.658 & 0.014 & 1.091 & 0.012 & 0.523 & 0.014 & 7.017 & 0.089 & 4.440 & 0.052 & 2.577 & 0.074 \\
\hline
\end{tabular}


TABLE III. (Continued).

\begin{tabular}{|c|c|c|c|c|c|c|c|c|c|c|c|c|}
\hline \multirow[b]{2}{*}{$E$} & \multicolumn{6}{|c|}{ Neon } & \multicolumn{6}{|c|}{ Argon } \\
\hline & $\sigma_{G T}$ & Error & $\sigma_{G T-P s}$ & Error & $\sigma_{P s}$ & Error & $\sigma_{G T}$ & Error & $\sigma_{G T-P s}$ & Error & $\sigma_{P s}$ & Error \\
\hline 30.000 & 1.716 & 0.011 & 1.137 & 0.009 & 0.531 & 0.011 & 7.120 & 0.088 & 4.516 & 0.051 & 2.604 & 0.072 \\
\hline 31.000 & 1.738 & 0.011 & 1.164 & 0.009 & 0.534 & 0.010 & 7.157 & 0.082 & 4.612 & 0.053 & 2.546 & 0.071 \\
\hline 32.000 & 1.772 & 0.011 & 1.211 & 0.010 & 0.517 & 0.010 & 7.205 & 0.090 & 4.711 & 0.051 & 2.494 & 0.074 \\
\hline 33.000 & 1.799 & 0.016 & 1.253 & 0.015 & 0.490 & 0.016 & 7.197 & 0.085 & 4.732 & 0.052 & 2.466 & 0.073 \\
\hline 34.000 & 1.792 & 0.016 & 1.239 & 0.014 & 0.489 & 0.016 & 7.258 & 0.087 & 4.895 & 0.049 & 2.364 & 0.076 \\
\hline 35.000 & 1.816 & 0.016 & 1.271 & 0.015 & 0.499 & 0.016 & 7.203 & 0.086 & 4.886 & 0.053 & 2.317 & 0.073 \\
\hline 36.000 & 1.826 & 0.017 & 1.295 & 0.015 & 0.471 & 0.016 & 7.268 & 0.082 & 4.939 & 0.052 & 2.329 & 0.071 \\
\hline 37.000 & 1.854 & 0.017 & 1.335 & 0.014 & 0.470 & 0.016 & 7.101 & 0.087 & 4.979 & 0.052 & 2.122 & 0.070 \\
\hline 38.000 & 1.835 & 0.009 & 1.296 & 0.008 & 0.471 & 0.009 & 7.366 & 0.083 & 5.113 & 0.048 & 2.253 & 0.070 \\
\hline 39.000 & 1.855 & 0.010 & 1.340 & 0.008 & 0.467 & 0.009 & 7.303 & 0.082 & 5.118 & 0.045 & 2.185 & 0.070 \\
\hline 40.000 & 1.867 & 0.009 & 1.360 & 0.007 & 0.454 & 0.009 & 7.276 & 0.088 & 5.167 & 0.052 & 2.109 & 0.072 \\
\hline 41.000 & 1.855 & 0.010 & 1.356 & 0.008 & 0.443 & 0.010 & 7.186 & 0.083 & 5.126 & 0.048 & 2.060 & 0.072 \\
\hline 42.000 & 1.871 & 0.010 & 1.390 & 0.008 & 0.428 & 0.010 & 7.308 & 0.080 & 5.283 & 0.054 & 2.025 & 0.069 \\
\hline 43.000 & 1.870 & 0.010 & 1.390 & 0.009 & 0.430 & 0.010 & 7.292 & 0.083 & 5.308 & 0.051 & 1.984 & 0.071 \\
\hline 44.000 & 1.863 & 0.010 & 1.398 & 0.008 & 0.414 & 0.010 & 7.303 & 0.084 & 5.385 & 0.051 & 1.919 & 0.070 \\
\hline 45.000 & 1.886 & 0.010 & 1.418 & 0.008 & 0.402 & 0.010 & 7.220 & 0.081 & 5.403 & 0.048 & 1.816 & 0.068 \\
\hline 46.000 & 1.864 & 0.010 & 1.420 & 0.008 & 0.393 & 0.010 & 7.276 & 0.084 & 5.481 & 0.053 & 1.795 & 0.068 \\
\hline 47.000 & 1.885 & 0.010 & 1.453 & 0.008 & 0.393 & 0.010 & 7.213 & 0.080 & 5.439 & 0.048 & 1.774 & 0.066 \\
\hline 48.000 & 1.889 & 0.010 & 1.454 & 0.008 & 0.385 & 0.010 & 7.109 & 0.079 & 5.372 & 0.050 & 1.737 & 0.069 \\
\hline 49.000 & 1.881 & 0.010 & 1.478 & 0.008 & 0.368 & 0.010 & 7.107 & 0.080 & 5.470 & 0.051 & 1.638 & 0.065 \\
\hline 50.000 & 1.886 & 0.010 & 1.479 & 0.008 & 0.369 & 0.010 & 7.308 & 0.081 & 5.575 & 0.048 & 1.734 & 0.067 \\
\hline 51.000 & 1.897 & 0.010 & 1.497 & 0.008 & 0.367 & 0.010 & 7.165 & 0.080 & 5.557 & 0.047 & 1.608 & 0.064 \\
\hline 52.000 & 1.904 & 0.010 & 1.527 & 0.008 & 0.354 & 0.010 & 7.022 & 0.083 & 5.446 & 0.053 & 1.576 & 0.065 \\
\hline 53.000 & 1.887 & 0.010 & 1.511 & 0.008 & 0.338 & 0.010 & 7.165 & 0.078 & 5.603 & 0.049 & 1.561 & 0.064 \\
\hline 54.000 & 1.903 & 0.010 & 1.523 & 0.009 & 0.341 & 0.010 & 7.085 & 0.077 & 5.613 & 0.049 & 1.472 & 0.065 \\
\hline 55.000 & 1.905 & 0.010 & 1.537 & 0.008 & 0.333 & 0.010 & 7.117 & 0.076 & 5.671 & 0.049 & 1.446 & 0.064 \\
\hline 56.000 & 1.909 & 0.010 & 1.560 & 0.008 & 0.309 & 0.009 & 7.027 & 0.078 & 5.620 & 0.049 & 1.406 & 0.061 \\
\hline 57.000 & 1.894 & 0.010 & 1.550 & 0.009 & 0.316 & 0.010 & 6.903 & 0.072 & 5.576 & 0.049 & 1.327 & 0.061 \\
\hline 58.000 & 1.898 & 0.010 & 1.570 & 0.009 & 0.290 & 0.009 & 7.089 & 0.076 & 5.697 & 0.050 & 1.392 & 0.059 \\
\hline 59.000 & 1.895 & 0.010 & 1.581 & 0.009 & 0.283 & 0.009 & 6.957 & 0.073 & 5.702 & 0.052 & 1.255 & 0.057 \\
\hline 60.000 & 1.898 & 0.009 & 1.592 & 0.009 & 0.283 & 0.008 & 7.069 & 0.070 & 5.778 & 0.048 & 1.291 & 0.052 \\
\hline
\end{tabular}

The present measurements of $\sigma_{P s}$ in neon are broadly consistent with the previous results of Marler et al. and Laricchia et al. Differences between the present result and those of Marler et al. are speculated to arise from the latter authors' use of the single-scattering approximation. Theoretical treatment of the Ps formation cross section fails to successfully reproduce either the magnitude or energy dependence of the cross section presently found, and suggests that a more sophisticated treatment of the problem is required.

In $\mathrm{Ar}$, below $E_{P S}$, the present $\sigma_{G T}$ result deviates substantially from the majority of previous measurements, although there is reasonable agreement between the present results and those of Canter et al. and Jaduszliwer and Paul. Similarly, most of the theoretical approaches considered, including the present ROP calculation used in correcting the present experimental data, lie below the present results between 2 and $9 \mathrm{eV}$, with only the work of Dzuba et al. and the present CCC calculation predicting a cross section of comparable magnitude. Above $E_{P s}$ there is better consensus among different measurements and the present results. The measurement of the total cross section between 20 and $60 \mathrm{eV}$ greatly improves the density of experimental results in this range and allows a more critical analysis of theoretical calculations. The only reasonable agreement with theory is found in the present CCC calculations, with other predictions overestimating the cross section above $20 \mathrm{eV}$. Comparison of the present measurements with the present ROP calculations above $E_{P_{S}}$ is less satisfactory in Ar than that found in $\mathrm{Ne}$, with the present ROP prediction of $\sigma_{G T-P s}$ approaching a value at $60 \mathrm{eV}$ that is larger than the present measurement of $\sigma_{G T}$.

The Ps formation cross section in argon shows a similar level of disagreement with the measurements of Marler et al., again accounted for primarily by their use of the singlescattering approximation. The present measurements shows no sign of the second peak observed in the data of Laricchia et al.

\section{ACKNOWLEDGMENTS}

The authors would like to acknowledge the Australian Researh Council's (ARC) Centre of Excellence Program for providing funding. One of the authors, C.M., is grateful to the ARC for financial support. We would also like to thank the technical staff, Graeme Cornish, Stephen Battisson, Ross Tranter, and Ron Cruikshank, for their frequent assistance and technical advice. 
[1] M. Charlton, Rep. Prog. Phys. 48, 737 (1985).

[2] C. Makochekanwa, A. Bankovic, W. Tattersall, A. Jones, P. Caradonna, D. S. Slaughter, K. Nixon, M. J. Brunger, Z. Petrovic, J. P. Sullivan, and S. J. Buckman, New J. Phys. 11, 103036 (2009).

[3] J. P. Sullivan, A. Jones, P. Caradonna, C. Makochekanwa, and S. J. Buckman, Rev. Sci. Instrum. 79, 113105 (2008).

[4] J. P. Sullivan, S. J. Gilbert, J. P. Marler, R. G. Greaves, S. J. Buckman, and C. M. Surko, Phys. Rev. A 66, 042708 (2002).

[5] P. Caradonna, A. Jones, C. Makochekanwa, D. S. Slaughter, J. P. Sullivan, S. J. Buckman, I. Bray, and D. V. Fursa, Phys. Rev. A 80, 032710 (2009).

[6] J. P. Sullivan, C. Makochekanwa, A. Jones, P. Caradonna, D. S. Slaughter, J. Machacek, R. P. McEachran, D. W. Mueller, and S. J. Buckman, J. Phys. B 44, 035201 (2011).

[7] R. P. McEachran, A. G. Ryman, and A. D. Stauffer, J. Phys. B 11, 551 (1978).

[8] R. P. McEachran, A. G. Ryman, and A. D. Stauffer, J. Phys. B 12, 1031 (1979).

[9] S. Chen, R. P. McEachran, and A. D. Stauffer, J. Phys. B 41, 025201 (2008).

[10] R. P. McEachran and A. D. Stauffer, J. Phys. B 23, 4605 (1990).

[11] I. P. Grant, B. J. McKenzie, P. H. Norrington, D. F. Mayers, and N. C. Pyper, Comput. Phys. Commun. 21, 207 (1980).

[12] R. P. McEachran and A. D. Stauffer, J. Phys. B 42, 075202 (2009).

[13] I. Bray and A. T. Stelbovics, Phys. Rev. A 48, 4787 (1993).

[14] H. Wu, I. Bray, D. V. Fursa, and A. T. Stelbovics, J. Phys. B 37, L1 (2004).

[15] H. Wu, I. Bray, D. V. Fursa, and A. T. Stelbovics, J. Phys. B 37, 1165 (2004).

[16] D. V. Fursa and I. Bray, Phys. Rev. A 52, 1279 (1995).

[17] R. P. Saxon, J. Chem. Phys. 59, 1539 (1973).

[18] R. R. Teachout and R. T. Pack, At. Data Nucl. Data Tables 3, 195 (1971).

[19] P. G. Coleman, T. C. Grith, G. R. Heyland, and T. R. Twomey, Appl. Phys. 11, 321 (1976).

[20] H. Nakanishi and D. M. Schrader, Phys. Rev. A 34, 1823 (1986).

[21] K. L. Baluja and A. Jain, Phys. Rev. A 46, 1279 (1992).
[22] V. A. Dzuba, V. V. Flambaum, G. F. Gribakin, and W. A. King, J. Phys. B 29, 3151 (1996).

[23] K. F. Canter, P. G. Coleman, T. C. Grith, and G. R. Heyland, J. Phys. B 6, L201 (1973).

[24] B. Jaduszliwer and D. A. L. Paul, Appl. Phys. 3, 281 (1974).

[25] T. S. Stein, W. E. Kauppila, V. Pol, J. H. Smart, and G. Jesion, Phys. Rev. A 17, 1600 (1978).

[26] G. Sinapius, W. Raith, and W. G. Wilson, J. Phys. B 13, 4079 (1980).

[27] M. Charlton, G. Laricchia, T. C. Grith, G. L. Wright, and G. R. Heyland, J. Phys. B 17, 4945 (1984).

[28] P. G. Coleman, J. D. McNutt, L. M. Diana, and J. R. Burciaga, Phys. Rev. A 20, 145 (1979).

[29] W. E. Kauppila, T. S. Stein, J. H. Smart, M. S. Dababneh, Y. K. Ho, J. P. Downing, and V. Pol, Phys. Rev. A 24, 725 (1981).

[30] A. C. L. Jones, P. Caradonna, C. Makochekanwa, D. S. Slaughter, R. P. McEachran, J. R. Machacek, J. P. Sullivan, and S. J. Buckman, Phys. Rev. Lett. 105, 073201 (2010).

[31] G. Laricchia, P. Van Reeth, M. Szluinska, and J. Moxom, J. Phys. B 35, 2525 (2002).

[32] J. P. Marler, J. P. Sullivan, and C. M. Surko, Phys. Rev. A 71, 022701 (2005).

[33] M. T. McAlinden and H. R. J. Walters, Hyperfine Interact. 73, 65 (1992).

[34] S. Gilmore, J. E. Blackwood, and H. R. J. Walters, Nucl. Instrum. Methods Phys. Res. Sect. B 221, 129 (2004).

[35] R. E. Montgomery and R. W. LaBahn, Can. J. Phys. 48, 1288 (1970).

[36] A. Jain, Phys. Rev. A 41, 2437 (1990).

[37] S. N. Nahar and J. M. Wadehra, Phys. Rev. A 43, 1275 (1991).

[38] W. E. Kauppila, T. S. Stein, and G. Jesion, Phys. Rev. Lett. 36, 580 (1976).

[39] G. P. Karwasz, D. Pliszka, and R. S. Brusa, Nucl. Instrum. Methods Phys. Res. Sect. B 247, 68 (2006).

[40] P. G. Coleman, J. D. McNutt, L. M. Diana, and J. T. Hutton, Phys. Rev. A 22, 2290 (1980).

[41] A. Jones, P. Caradonna, C. Makochekanwa, D. Slaughter, D. Mueller, J. P. Sullivan, and S. J. Buckman, J. Phys. Conf. Ser. 194, 012033 (2009). 